\title{
Building up cyclodextrins from scratch - templated enzymatic synthesis of cyclodextrins directly from maltose
}

\author{
Larsen, Dennis; Beeren, Sophie
}

Published in:

Chemical Communications

Link to article, DOI:

10.1039/d1cc00137j

Publication date:

2021

Document Version

Peer reviewed version

Link back to DTU Orbit

Citation (APA):

Larsen, D., \& Beeren, S. (2021). Building up cyclodextrins from scratch - templated enzymatic synthesis of cyclodextrins directly from maltose. Chemical Communications, 57, 8456-8460.

https://doi.org/10.1039/d1cc00137]

\section{General rights}

Copyright and moral rights for the publications made accessible in the public portal are retained by the authors and/or other copyright owners and it is a condition of accessing publications that users recognise and abide by the legal requirements associated with these rights.

- Users may download and print one copy of any publication from the public portal for the purpose of private study or research.

- You may not further distribute the material or use it for any profit-making activity or commercial gain

- You may freely distribute the URL identifying the publication in the public portal

If you believe that this document breaches copyright please contact us providing details, and we will remove access to the work immediately and investigate your claim. 


\title{
Building up cyclodextrins from scratch - templated enzymatic synthesis of cyclodextrins directly from maltose
}

Received 00th January 20xx, Accepted 00th January 20xx

\author{
Dennis Larsen ${ }^{\mathrm{a}}$ and Sophie R. Beeren*a
}

DOI: $10.1039 / \times 0 \times x 00000 x$

Cyclodextrins (CDs) are commercially produced via enzymatic breakdown of starch or amylose. In contrast, we show that cyclodextrins can be synthesised directly from the disaccharide maltose in good yields by exploiting the use of templates to favour the enzymatic build-up of cyclodextrins. Using cyclodextrin glucanotransferase to catalyse reversible transglycosylation, and 1adamantane carboxylic acid as the template, we can synthesise $\beta$ CD from maltose in approximately $70 \%$ yield. This work represents a step towards supramolecular control over enzymatic production of complex oligosaccharides from simple building blocks.

The efficient production of oligosaccharides continues to be a challenge for the synthetic chemistry community. ${ }^{1}$ Stepwise syntheses, involving sequential protection, glycosylation and deprotection, can be impressive, but require highly-skilled synthetic chemists and are rarely scalable, and thus only a few therapeutically important oligosaccharides are produced synthetically on an industrial scale. ${ }^{2}$ In contrast, other biopolymers, oligonucleotides and proteins, are readily assembled using a combination of automation (e.g. DNA synthesis) and biotechnologies that employ templatecontrolled enzymatic synthesis (e.g. PCR and protein expression). ${ }^{3}$ Chemoenzymatic and automated approaches to oligosaccharide synthesis that harness the unique capacity of enzymes to control chemoselectivity and stereoselectivity have shown recent success. ${ }^{4}$ We are seeking to develop an approach that exploits enzymes to generate dynamic mixtures of oligosaccharides that interconvert via the reversible formation of glycosidic linkages, and employs synthetic templates to bind, select and amplify specific oligosaccharide products.

Cyclodextrins (CDs) are macrocyclic oligosaccharides that exhibit truncated cone-like structures made up of typically 6,7

\footnotetext{
a. Department of Chemistry, Technical University of Denmark, DK-2800 Kongens Lyngby, Denmark.

* To whom correspondence should be addressed: sopbee@kemi.dtu.dk.

Electronic Supplementary Information (ESI) available: [experimental details, HPLCELS chromatograms, NMR spectra, reaction monitoring]. See DOI: $10.1039 / \times 0 \times x 00000 x$
}

or $8(\alpha, \beta$, or $\gamma$-CD) D-glucopyranose units linked by $\alpha-1,4$ glycosidic bonds. Due to the relatively hydrophobic nature of the $C D$ cavities, and their concomitant ability to bind and the $C D$ cavities, and their concomitant ability to bind and increase the aqueous solubility of poorly soluble compounds, they have found wide applicability within many fields, such as in the cosmetics, pharmaceutics, and food industries. ${ }^{5}$ Commercially available CDs are produced by treating biopolymers made up of D-glucopyranose units linked by $\alpha-1,4$-glycosidic bonds $(\alpha-1,4-$ glucans), such as starch or amylose, with cyclodextrin glucanotransferase (CGTase), which breaks down the biopolymer into primarily CDs and small amounts of linear oligosaccharides. $^{6}$ The build-up of cyclodextrins from monosaccharides using synthetic organic chemistry has been described but these are complex multistep-syntheses that are impressive, but inevitably low yielding. ${ }^{7}$

We have recently shown how the process by which CGTase makes CDs from an $\alpha$-1,4-glucan source is dynamic and responsive. ${ }^{8}$ The enzyme scrambles the glycosidic linkages in linear and cyclic $\alpha-1,4-$ glucans enabling the interconversion of CDs via a series of transglycosylation steps. The CDs in this dynamic system form as a kinetically-trapped subsystem that exists transiently before the eventual conversion of all glucan material to glucose (G1), the thermodynamic product. ${ }^{8 a}$ The CDs exist in what is essentially a transient Dynamic Combinatorial Library (DCL), and can interconvert via enzyme-mediated dynamic covalent chemistry. Using template molecules, we can control the selective interconversion of CDs. For example, we could successfully turn $\alpha-C D$ into $\beta-C D$ by treating $\alpha-C D$ with CGTase in the presence of 1-adamantane carboxylic acid, a compound that binds strongly and selectively to $\beta-\mathrm{CD} .{ }^{8 a}$

In the present communication we show that by exploiting template effects, CDs can be synthesised directly from maltose (G2) (Fig. 1). Our approach of building up CDs from small building blocks is the reverse process of the polymer breakdown used in current CD production, and represents a step towards supramolecular control over enzymatic production of complex oligosaccharides from simple carbohydrate building blocks. 
a) This study

Making CDs from disaccharide building blocks:

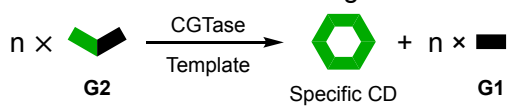

Previous studies

Making CDs by polymer breakdown:

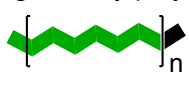

$\alpha-1,4$-glucan (e.g. amylose)

b)
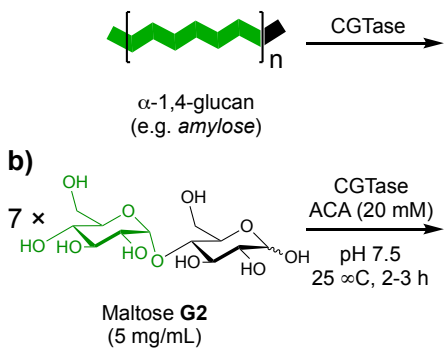

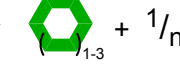

Mix of CDs

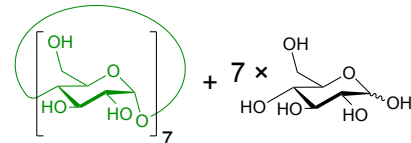

Glucose $\mathbf{G 1}$
G1

1

$$
\text { yield: } 70 \%
$$

Fig. 1. a) Concept: synthesis of specific CDs by CGTase-catalysed build-up from the shortest $\alpha$-1,4-glucan oligomer, maltose (G2) in the presence of a template in contrast to the commercial production of CDs by CGTase-catalysed breakdown of $\alpha-1,4$-glucan polymers. b) $\beta-C D$ can be enzymatically synthesised from $\mathbf{G 2}$ in $70 \%$ yield by using adamantane carboxylic acid (ACA) as a template.

Maltose (G2), and not glucose (G1), is the simplest building block from which CDs can be assembled by CGTase. CGTase scrambles $\alpha-1,4$-glycosidic linkages in a two-step process. ${ }^{9}$ Firstly, a covalent enzyme-substrate glycosyl donor complex is formed by cleaving one $\alpha-1,4$-glycosidic linkage in the substrate, and if the substrate is non-macrocyclic, releasing D-glucose (G1) or a longer $\alpha$-1,4-glucan. Secondly, a new $\alpha$-1,4-glycosidic linkage is formed when the enzyme is displaced by a glycosyl acceptor. If this second step involves an intramolecular reaction, a CD is formed, and if it is intermolecular a new linear $\alpha-1,4$-glucan is formed. In the case of $\mathbf{G 2}$ as a substrate, the reducing end (hemiacetal) D-glucopyranose unit (illustrated with black rectangles in Fig. 1a can thus be considered a "leaving group." To form 1 mole of $\alpha-C D, 6$ moles of $\mathbf{G 2}$ would thus be required and 6 moles of $\mathbf{G 1}$ are generated as a by-product (see section $\mathrm{S} 2, \mathrm{ESI})$.

To investigate whether or not the inexpensive, readily available, and highly soluble maltose (G2) could be utilised to synthesise CDs, we used NMR spectroscopy. CGTase $(0.57$ $\mathrm{mg} / \mathrm{mL})$ was added to a solution of $\mathbf{G 2}(10 \mathrm{mg} / \mathrm{mL})$ in $D_{2} \mathrm{O}$ buffered with sodium phosphate $\left(50 \mathrm{mM}, \mathrm{pH} \mathrm{7.5)}\right.$ at $25^{\circ} \mathrm{C}$. The relative total concentration of $\mathrm{CDs}$ was quantified by comparing the integrals for anomeric $(\mathrm{H} 1)$ signals of the $\mathrm{CDs}\left(\mathrm{H} 1_{\mathrm{CDs}}\right.$, highlighted in blue in Fig. 2a, 5.06-4.96 ppm), the reducing-end (hemiacetal) $\alpha$-anomer of the linear oligomers $\left(\alpha-\mathrm{H} 1_{\text {red.ends, }}\right.$ highlighted in yellow, $5.18-5.13 \mathrm{ppm}$ ) and the $\alpha-1 \rightarrow 4$ linked internal (acetal) units of the linear oligomers $\left(H 1_{\text {internal, }}\right.$ highlighted in red, $5.36-5.28$ ppm) (Fig. 2a). Some CD formation was observed, but the conversion was poor, and a maximum molar yield of $10 \%$ was obtained after $1.5-2$ hours. We report the molar CD yield taking into consideration that only acetal glucopyranose units can be incorporated into $\mathrm{CDs}$. Therefore, molar yield $=100 \% \times(6[\alpha-C D]+7[\beta-C D]+8[\gamma-C D]) /$ $\left((n-1)\left[G_{n}\right]_{0}\right)$ where $\left[G_{n}\right]_{\circ}$ is the concentration of the linear starting material used in the reaction and $n$ is the number of Dglucopyranose units in the starting material (i.e. $n=2$ for G2).

a)
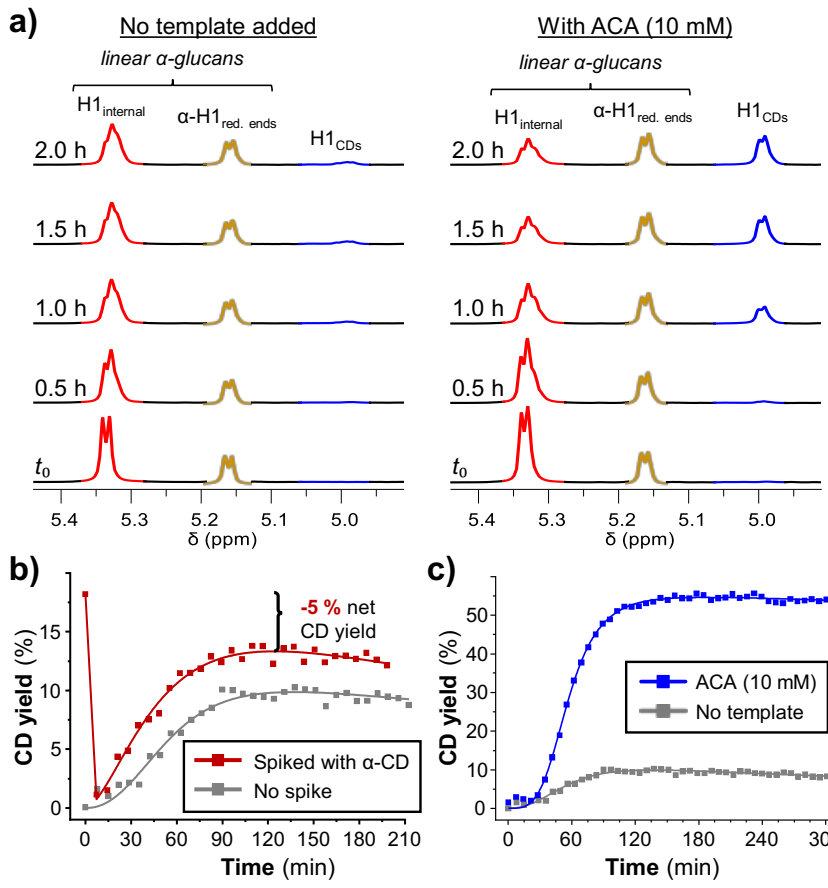

c)

Fig. 2. $C D$ yields obtained from $\mathbf{G} 2$ in presence of different templates as quantified by ${ }^{1} \mathrm{H}$ NMR spectroscopy. a) Partial ${ }^{1} \mathrm{H}$ NMR spectra during the reaction of $\mathbf{G 2}(10 \mathrm{mg} / \mathrm{mL})$ in sodium phosphate buffer $(50 \mathrm{mM}, \mathrm{pH} 7.5)$ with CGTase $(0.57 \mathrm{mg} / \mathrm{mL})$ at $25{ }^{\circ} \mathrm{C}$ in the absence and presence of ACA (10 mM). The coloured regions of the spectra correspond to the peak for the similarly coloured protons highlighted in the general structures for $\alpha$ glucans and CDs (box). b) Plot of calculated $C D$ yields ( $\left.{ }^{1} \mathrm{H} N M R\right)$ vs. time with and without $\alpha-C D$ spike and $c$ ) in presence and absence of ACA.

The relative concentrations of $\alpha, \beta$, and $\gamma$-CD were not determined at this point, as their signals are not sufficiently well resolved using ${ }^{1} \mathrm{H} N M R$, but from previous experiments we expected all three would form. ${ }^{8 a, c}$

CD production from $\mathbf{G 2}$ did not start immediately (Fig. 2b, grey trace). We observed an initial lag-phase, which can be explained by the fact that a number of intermolecular transglycosylation steps are required in order to build up linear $\alpha$-glucans that are long enough to produce $\mathrm{CDs}$ via an intramolecular transglycosylation. At least 6 transglycosylation steps are required to convert $\mathbf{G 2}$ to $\alpha-\mathrm{CD}$ (see section S2, ESI). The build-up of longer $\alpha$-glucans during the first 30 mins is seen in the broadening of the $\mathrm{H} 1$ signal from the internal $\alpha-1-4$ linked D-glucopyranose units $(5.36-5.28 \mathrm{ppm})$, due to the presence of several overlapping signals (Fig. 2a, left).

We initially thought that a more efficient conversion of $\mathbf{G 2}$ to $C D s$ could perhaps be achieved by 'spiking' our reactions with small amounts of longer linear $\alpha$-glucans or CDs in order to reduce the number of transglycosylation steps required to convert the $\mathbf{G} 2$ to CDs. $\mathbf{G} 2(9 \mathrm{mg} / \mathrm{mL})$ was thus treated with CGTase in the presence of $\alpha-C D(1 \mathrm{mg} / \mathrm{mL})$ (Fig. $2 \mathrm{~b}$ and section $\mathrm{S3}, \mathrm{ESI})$. Although the lag-phase was indeed shortened by 'spiking' the reaction with $\alpha-C D$, the large excess of short glycosyl acceptors rapidly converted the $\alpha-C D$ to short linear oligosaccharides resulting in a CD yield increase that was lower than the amount of CD spike (section S3, ESI).

Having previously shown that templates can promote the selective synthesis of $\mathrm{CDs}$ from maltohexaose (G6) by forming 
specific stabilising host-guest complexes, ${ }^{8 a}$ we hypothesised that higher conversion of $\mathbf{G} 2$ to $\mathrm{CDs}$ might be promoted by addition of a template. $\mathbf{G} 2(10 \mathrm{mg} / \mathrm{ml})$ was therefore treated with CGTase $(0.57 \mathrm{mg} / \mathrm{mL})$ in the presence of sodium dodecyl sulfate (SDS) (5 mM), 1-adamantane carboxylic acid (ACA) $(10 \mathrm{mM})$ or sodium tetraphenylborate $\left(\mathrm{NaBPh}_{4}\right)(10 \mathrm{mM})$, known to bind $\alpha-C D, \beta-C D$ and $\gamma-C D$, respectively. In addition to reaction monitoring by ${ }^{1} \mathrm{H}$ NMR spectroscopy, high performance liquid chromatography (HPLC) coupled to an evaporative light scattering (ELS) detector was utilised in order to ascertain the specific CD composition formed. Calibration curves from $0.01 \mathrm{mg} / \mathrm{mL}$ to $10 \mathrm{mg} / \mathrm{mL}$ for all three CDs as well as the linear $\alpha-1,4$-glucans $\mathbf{G 1}$ to $\mathbf{G 8}$ were made in order to correct for differences in the response of ELS detector to different oligosaccharides (S1, ESI).

In the presence of templates, we saw remarkably high levels of CD synthesis from G2 (Fig. 2a,b and Figs. S12, S13, ESI). The yields and distribution of $C D$ products are shown in Figure $3 a$. Most impressively, $\beta-C D$ was synthesised from $\mathbf{G 2}$ in $58 \pm 4 \%$ yield in 3 hours with $>95 \%$ selectivity using ACA as a template. ${ }^{10}$ To optimise the selective synthesis of $\beta$-CD from G2, we examined the influence of template concentration and starting G2 concentration. By increasing the concentration of ACA template from $10 \mathrm{mM}$ to $20 \mathrm{mM}$, the yield increased to $67 \pm 2 \%$ (Fig. 3b). By decreasing the starting concentration of $\mathbf{G 2}$ to $5 \mathrm{mg} / \mathrm{mL}$ we obtained a further improved yield of $70 \pm 3 \%$ in the presence of $20 \mathrm{mM}$ ACA (Figs 1b, 3c and Fig. S14, ESI). We propose that the increased conversion of $\mathbf{G 2}$ to $\mathrm{CDs}$ in the presence of templates is due to a kinetically controlled "capture" mechanism. As soon as CDs are formed, they are bound by the templates, thus stabilising them and preventing their conversion back to short linear $\alpha-1,4$-glucans, and ultimately glucose.

In a true dynamic combinatorial library operating under thermodynamic control, the product distribution at equilibrium should be dependent only on the available building blocks and not on the specific starting material. In contrast, since this dynamic system forms CDs out-of-equilibrium in a kinetic trap, the choice of starting material is significant. It is noteworthy that the highest yield of CDs from $\mathbf{G 2}$ in the absence of templates is $10 \%$ while we have previously observed yields of over $60 \%$ for the same reaction started from G6. To better understand this effect, each of the linear $\alpha$-1,4-glucans ranging from maltose (G2) up to maltooctaose (G8) was treated with CGTase in the absence of template and the reactions monitored over time using HPLC-ELS (Fig. 4a,b and Figs. S15 - S17, ESI).

The molar CD yield was found to increase, and the time to reach maximum $C D$ yield to decrease, as the length of the oligosaccharide starting material was increase (Fig. 4a). There appears to be an inverse correlation between the CD yield and the number of transglycosylation steps required to form CDs from the starting material, making $\mathbf{G} 2$ the most challenging substrate. In all cases it was observed that once formed, a steady concentration of medium length (G4-G6) and long (G7 and G8) $\alpha$-1,4-glucans is observed, while CDs are first built up and then slowly converted into the short linear $\alpha-1,4$-glucans (G1-G3) (Fig. 4b). For G2-G6, which must first undergo at least a

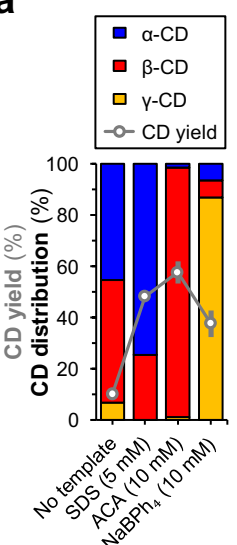

b
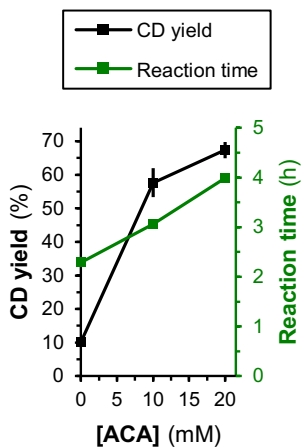

C
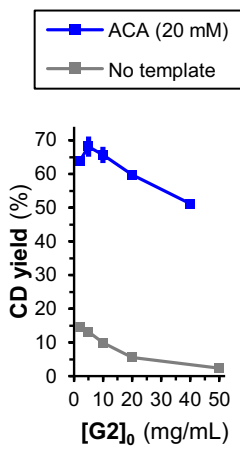

Fig. 3. a) Molar CD yields and CD composition (HPLC-ELS) obtained in reaction of G2 (10 $\mathrm{mg} / \mathrm{mL}$ ) with CGTase in the presence and absence of templates. b) Dependence of molar $C D$ yield and reaction time on ACA concentration. c) Plot showing the influence of $\mathbf{G 2}$ starting concentration on molar CD yield with and without ACA (20 mM). Conditions otherwise as in Fig. 2.10

one intermolecular transglycosylation to form a longer linear $\alpha$ 1,4-glucan before cyclisation to form CDs is possible, we expect a lag-phase before CDs begin to form. This is not immediately evident from Fig. 4b for $\mathbf{G 4}$ and $\mathbf{G 6}$, but can in fact be observed for $\mathbf{G 6}$ when the reaction is analysed with very high time resolution (Fig. S18, ESI). In contrast, when the reaction is started from $\mathbf{G 7}$ and $\mathbf{G 8}, \mathrm{CDs}$ can form directly, resulting in higher quantities of $\alpha$-glucan material being kinetically trapped as $C D$ s and therefore the higher $C D$ yields.

To summarise the workings of the system we propose the generalised energy diagram depicted in Fig. 4c. The short linear $\alpha$-glucans (G1-G3) represent the thermodynamically more stable products (with $\mathbf{G 1}$ being the final product upon reaction ad infinitum), ${ }^{8 a}$ while the CDs exist in a kinetically trapped subsystem. Exchange of D-glucopyranose building blocks between these two extremes must go via the medium length (G4-G6) and long (G>6) linear $\alpha$-glucans, which therefore represent higher energy intermediates. Long linear $\alpha$-glucans are only ever observed in very low concentrations, as they can readily convert to CDs (Fig 4b). Upon addition of templates an additional, deeper kinetic trap is formed, which significantly boosts the CD yields, even when starting from G2. Meanwhile addition of a template to the reaction starting from $\mathbf{G} 2$ does not affect the lag-phase since longer $\alpha$-1,4-glucans must first assemble before they can cyclise. The HPLC-ELSD data provide direct evidence of this, since the accumulation of higher quantities of kinetically-trapped CDs in the presence of template is first seen after the longer $\alpha$-glucans (G7 and G8) are detected in the system, i.e. after approximately 30 minutes of reaction (Fig. 4d).

In conclusion, we have developed a method to enzymatically build up cyclodextrins from maltose, in contrast to the conventional method which relies upon the breakdown of polysaccharides. In the absence of templates, this route is inefficient and low yielding. In combination with templates, dramatically improved CD yields can be obtained (up to ca. $70 \%$ in the case of the ACA template). The overall CGTase- mediated 
a)

b)
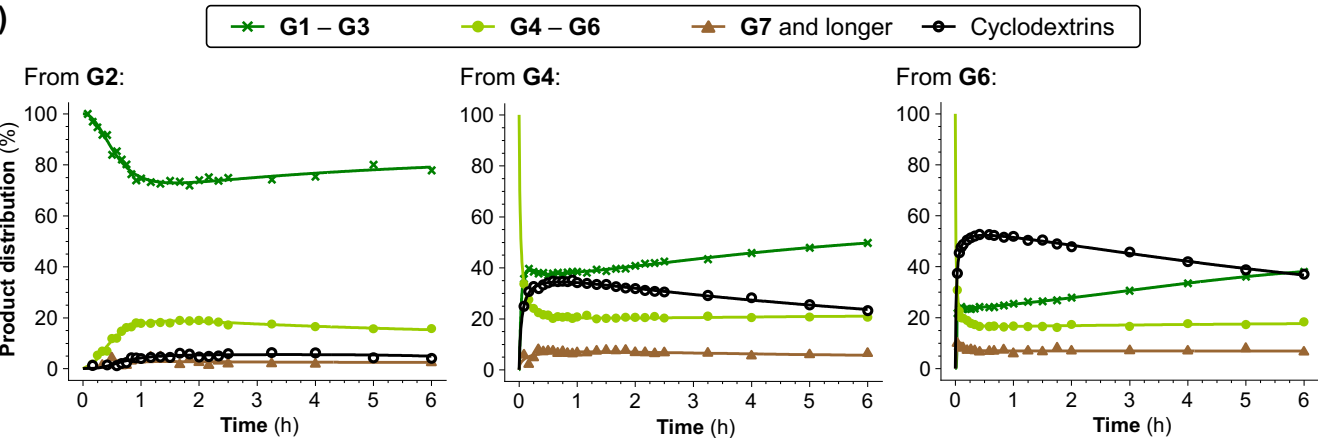

c)

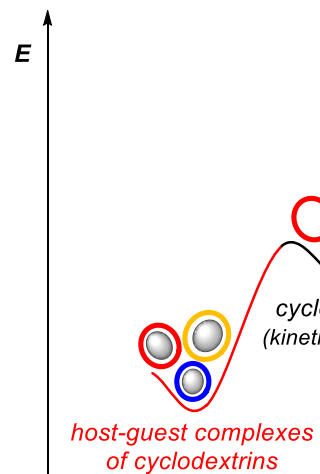

d)

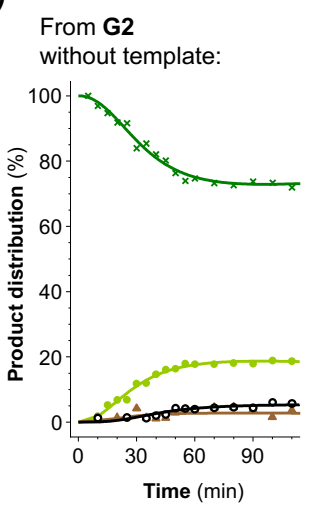

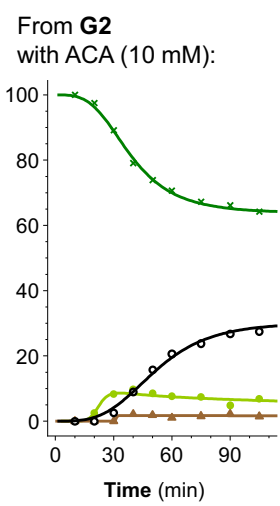

Fig. 4. a) Highest achieved molar CD yield and the reaction time needed to reach this yield as a function of starting material. b) Composition of reactions started from either $\mathbf{G 2}$, G4 or G6 (HPLC-ELS) showing higher CD yields and diminished lag-phases as the length of the starting $\alpha$-glucan increases (for from all starting materials G2 - G8, please see Fig. S15-17, ESI). c) Proposed energy diagram for the dynamic system afforded upon treating $\alpha$-glucans with CGTase in the presence of templates for cyclodextrins. $d$ ) Compositions of reactions from $\mathbf{G 2}$ in the absence and presence of ACA template (HPLC-ELS) over the first 100 minutes.

system of $\alpha$-glucans is dynamic but CDs are formed out-ofequilibrium, which means that the choice of starting material has an influence on the CD yield. Exploiting knowledge of this dynamic system allows us to use templates to kinetically trap $C D$ products that are otherwise formed transiently and sparingly, in order to favour their production profoundly. This system represents a first step towards the use of dynamic oligosaccharide systems to produce higher value and more complex oligosaccharides from simple building blocks by stabilising them with supramolecular interactions.

The authors are grateful to the Villum Foundation, the Carlsberg Foundation and the Novo Nordisk Foundation for financial support, and to Amano Enzyme, Inc., Nagoya, Japan, for kindly gifting us a stock solution of CGTase.

\section{Conflicts of interest}

There are no conflicts to declare.

\section{Notes and references}

1 (a) L. Krasnova and C.-H. Wong, J. Am. Chem. Soc., 2019, 141, 3735. (b) T. J. Boltje, T. Buskas and G.-J. Boons, Nat. Chem., 2009, 1, 611. (c) P. H. Seeberger and D. B. Werz, Nature, 2007, 446, 1046.

2 (a) X. Zhu and R. R. Schmidt, Angew. Chemie, 2009, 48, 1900. (b) O. J. Plante, E. R. Palmacci and P. H. Seeberger, Science,
2001, 291, 1523. (c) P.-A. Driguez, P. Potier and P. Trouilleux, Nat. Prod. Rep., 2014, 34, 980.

3 L. Wen, G. Garrett, C. Gibbons, J. Zhang, M. R. Gadi, H. Zhu, J. Fang, X. Liu, Y. Kong, R. G. Wang, Chem. Rev., 2018, 118, 8151.

4 (a) A. A. Joseph, A Pardo-Vargas and P. H. Seeberger, J. Am. Chem. Soc., 2020, 142, 8561. (b) T. Li, L. Liu, N. Wei, J.-Y. Yang, D. G. Chapla, K. W. Moremen, G.-J. Boons, Nat. Chem., 2019, 11, 229. (c) J. Zhang, C. Chen, M. R. Gadi, C. Gibbons, Y. Guo, X. Cao, G. Edmunds, S. Wang, D. Liu, J. Yu, L. Wen and P. G. Wang, Angew. Chemie Int. Ed., 2018, 57, 16638.

5 N. Sharma and A. Baldi, Drug. Deliv. 2016, 23, 729-747. E. M. M. Del Valle, Proc. Biochem., 2004, 39, 1033.

6 A. Biwer, G. Antranikian and E. Heinzle, 2002, 59, 609.

7 (a) D. Ikuta, Y. Hirata, S. Wakamori, H. Shimada, Y. Tomabechi, Y. Kawasaki, K. Ikeuchi, T. Hagimori, S. Matsumoto and H. Yamada, Science, 2019, 364, 674. (b) T. Nakagawa, K. Ueno, M. Kashiva and J. Watanabe, Tetrahedr. Lett., 1994, 35, 1921. (c) K. Maiti, G. C. Samantha, G. C. Daskhan and N. Jayaraman, Carbohydr. Chem., 2017, 42, 165.

8 (a) D. Larsen and S. R. Beeren, Chem. Sci., 2019, 10, 9981. (b) D. Larsen, P. M. Bjerre and S. R. Beeren, Chem. Commun., 2019, 55, 15037. (c) D. Larsen and S. R. Beeren, Chem. Eur. J., 2020, 26, 11032.

9 J. C. M. Uitdehaag, B. A. van der Veen, L. Dijkhuizen and B. W. Dijkstra, Enz. Microbiol. Techn., 2002, 30, 295.

10 Where the CD yield was determined by both NMR spectroscopy and HPLC-ELSD, it is reported as the average of the two determinations \pm one standard deviation. 